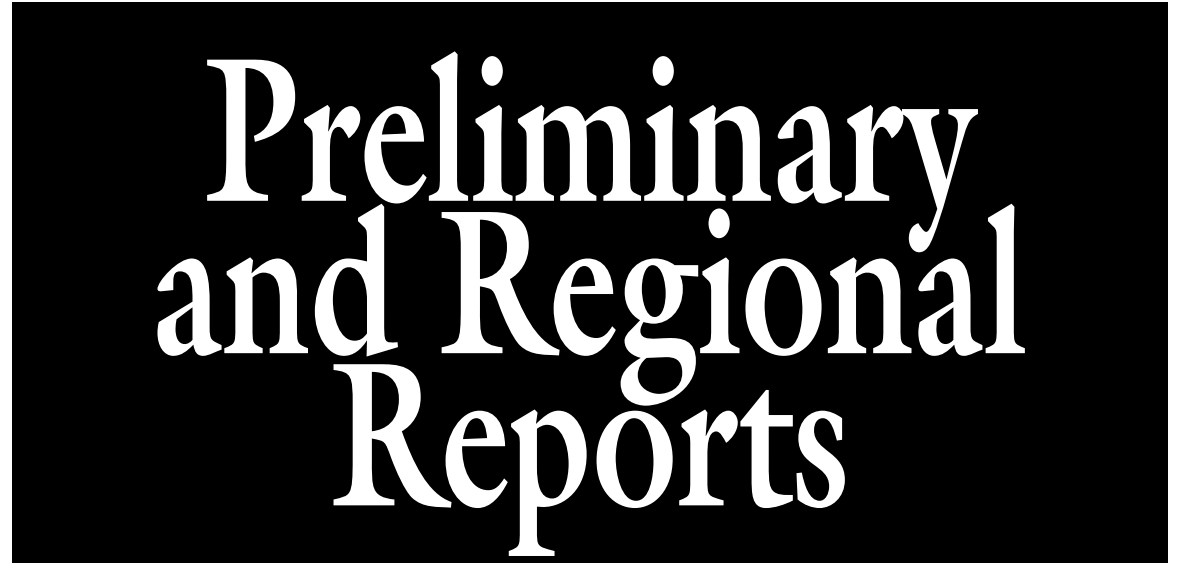

\section{Survey of 575 Daylily Cultivars for Severity of Daylily Rust in a Southern Mississippi Landscape}

\author{
Eugene K. Blythe ${ }^{1,4,7}$, Cecil Pounders ${ }^{2,5}$, Michael Anderson ${ }^{1,6}$, \\ Earl Watts ${ }^{3}$, and Barbara Watts ${ }^{3}$
}

AdDitional index words. Hemerocallis, Puccinia bemerocallidis, disease resistance, disease susceptibility

SUMMARY. Daylily (Hemerocallis sp.) is a popular and widely planted herbaceous perennial in the landscape, with over 78,000 cultivars registered with the American Hemerocallis Society. Daylily performs well in full sun, heat, humidity, and periods of dry weather, and has generally been considered to be pest free. However, a rust disease (Puccinia bemerocallidis) was introduced in the United States on imported plants in 2000, quickly spreading to become a widespread problem on daylily in and beyond the southern United States. In Aug. 2013, 575 daylily cultivars (mostly newer hybrids) were surveyed for daylily rust in a large landscape planting that had not received any fungicide treatment during the 2013 growing season. Weather conditions during the growing season were favorable for daylily rust. Individual clumps were rated as 1 (no or little visual sign of infection), 2 (moderate infection), or 3 (severe infection). In this survey, 119 cultivars (21\%) received a median rating of 1 or $1.5,230$ cultivars (40\%) received a rating of 2 , and $226(39 \%)$ received a rating of 2.5 or 3 . Most cultivars were represented by a single clump, and may thus be more susceptible to daylily rust than a single rating might indicate. Diploid cultivars were associated with lower daylily rust severity ratings than tetraploid cultivars.

Our thanks to Warren Copes, Hamidou Sakhanokho, and Donna Shaw for reviewing an early draft of this manuscript. This paper was approved for publication as Journal Article No. J-12659 of the Mississippi Agricultural and Forestry Experiment Station, Mississippi State University.

Mention of trade names or commercial products in this article is solely for the purpose of providing specific information and does not imply recommendation or endorsement to the exclusion of other products that may also be suitable.

${ }^{1}$ Coastal Research and Extension Center, Mississippi State University, South Mississippi Branch Experiment Station, P.O. Box 193, Poplarville, MS 39470

${ }^{2}$ USDA-ARS Thad Cochran Southern Horticultural Laboratory, P.O. Box 287, Poplarville, MS 39470

${ }^{3}$ Suburban Daylilies, 60 Serene Meadows Drive, Hattiesburg, MS 39402

${ }^{4}$ Associate Research Professor

${ }^{5}$ Research Plant Geneticist

${ }^{6}$ Research Associate

${ }^{7}$ Corresponding author. E-mail: blythe@pss.msstate.edu.

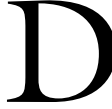
aylily is highly valued throughout the world as an easily grown herbaceous perennial for the landscape, with a proper selection of species and clonal hybrids providing an ongoing display of flowers from spring through fall. Daylily varies in growth habit; bloom season; and size, shape, and color of flowers. The robust and often fleshy roots of daylily withstand drought and their compact and sturdy, clumping growth habits allow daylily plants to tolerate competition from garden weeds (Hill and Hill, 1991; Stout, 1986). Daylily flowers may be used to flavor and garnish culinary dishes (Pollard et al., 2004).

Daylily generally has few insect and disease pests in the landscape; however, daylily rust, caused by the fungus Puccinia hemerocallidis, was observed for the first time in the United States on plants of the cultivar Pardon Me at a nursery in Georgia in Aug. 2000, apparently introduced via plants imported from South America (Williams-Woodward et al., 2001). Daylily rust spread quickly throughout much of the United States through movement of infected stock (Buck and Ono, 2012). Before 2000, the pathogen was known only from eastern Asia (Hernández et al., 2002). Multiple pathotypes of the pathogen have been identified in the southeastern United States (Buck, 2013). Disease management strategies include quarantine of new nursery stock, use of disease-resistant cultivars, scouting for signs and symptoms of rust, and use of preventative fungicides (Buck and Ono, 2012).

The daylily rust pathogen produces yellow-orange to rust-brown pustules containing urediniospores on upper and lower leaf surfaces within 1 to 2 weeks after infection (Williams-Woodward et al., 2001). Additional symptoms reported on infected plants include bright yelloworange spots and streaks, watersoaked tan spots with darker borders, large yellow lesions, and small discrete spots (Williams-Woodward and Buck, 2001). Mueller and Buck (2003) determined that temperatures around $72^{\circ} \mathrm{F}$ with $5 \mathrm{~h}$ of leaf wetness are required during infection; however, disease development is not as sensitive to environmental conditions once infection has taken place. Cultivar-specific resistance and partial resistance to the pathogen involve hypersensitive cell death and a delayed latent period and reduced sporulation resulting from restriction of intercellular hyphal growth (Li et al., 2007).

\begin{tabular}{llll}
\hline $\begin{array}{l}\text { Units } \\
\text { To convert U.S. to SI, } \\
\text { multiply by }\end{array}$ & U.S. unit & SI unit & $\begin{array}{l}\text { To convert SI to U.S., } \\
\text { multiply by }\end{array}$ \\
\hline 0.3048 & ft & $\mathrm{m}$ & 3.2808 \\
2.54 & inch $(\mathrm{es})$ & $\mathrm{cm}$ & 0.3937 \\
$\left({ }^{\circ} \mathrm{F}-32\right) \div 1.8$ & ${ }^{\circ} \mathrm{F}$ & ${ }^{\circ} \mathrm{C}$ & $\left({ }^{\circ} \mathrm{C} \times 1.8\right)+32$
\end{tabular}


Table 1. Daylily rust severity ratings on $\mathbf{5 7 5}$ daylily cultivars growing in a large landscape planting in southern Mississippi in Aug. 2013. Plants had not received any fungicide treatment during the 2013 growing season. Weather conditions during the growing season were favorable for daylily rust.

\begin{tabular}{|c|c|c|c|c|c|}
\hline Cultivar & Disease rating $^{z}$ & Bloom season $^{\mathrm{y}}$ & Winter foliage $^{\mathrm{x}}$ & Ploidy $^{\mathrm{w}}$ & Registration $^{v}$ \\
\hline Abilene Green Jeans & 1 & EM & $S$ & Tet & Maddox, 2010 \\
\hline Abilene Jiving Jessica & 2 & EM & $\mathrm{E}$ & Tet & Maddox, 2011 \\
\hline Across the Galaxy & 2 & M & $\mathrm{E}$ & Tet & Trimmer, 2007 \\
\hline Adorable Perfection & 1 & $\mathrm{E}$ & $\mathrm{E}$ & Dip & Carpenter-J., 2006 \\
\hline Aerial Display & 2 & $\mathrm{EE}$ & $\mathrm{E}$ & Tet & Stamile, 2003 \\
\hline Alabama Sweet Tee & 1 & M & $S$ & Dip & Herrington-K., 2009 \\
\hline Alex Sheets & 3 & EM & $S$ & Tet & Crochet, 2006 \\
\hline Alexander's Ragtime Band & 2.5 & EM & $\mathrm{E}$ & Tet & Kirchhoff-D., 1998 \\
\hline All About Eve & 1 & EM & $\mathrm{E}$ & Tet & Kirchhoff-D., 2000 \\
\hline Alpine Ruffles & 3 & M & $\mathrm{E}$ & Tet & Stamile, 2007 \\
\hline Amalia Harrison & 3 & EM & $S$ & Tet & George-T., 2007 \\
\hline Amber Rhum & 2 & M & $S$ & Tet & Petit, 2008 \\
\hline Annick Verhaert & 2 & $\mathrm{E}$ & $S$ & Tet & Kinnebrew-J., 2004 \\
\hline Appliqué & 2.5 & EM & $\mathrm{E}$ & Tet & Stamile, 2002 \\
\hline Apricot Cream Truffle & 1 & EM & $\mathrm{E}$ & Tet & Kirchhoff-D., 2007 \\
\hline Aquadisiac & 3 & EM & $\mathrm{E}$ & Tet & Harry, 2010 \\
\hline Artist Appliqué & 2 & $\mathrm{E}$ & $S$ & Tet & Lambertson, 2009 \\
\hline Ashes in My Eye & 3 & M & $S$ & Tet & Phelps, 2006 \\
\hline Asian Emissary & 3 & M & $S$ & Tet & Salter, 2003 \\
\hline Atomic Glow & 3 & M & $S$ & Tet & Smith-FR, 2007 \\
\hline Aunt Ethel & 3 & M & $S$ & Tet & Smith-FR, 2006 \\
\hline Aunt Joanne & 3 & M & $S$ & Tet & Smith-FR, 2006 \\
\hline Azure Wings & 2 & EM & $\mathrm{E}$ & Tet & Stamile, 2005 \\
\hline Baby Boomers Baby & 2 & EM & $\mathrm{D}$ & Dip & Herrington-T., 2003 \\
\hline Between Two Eternities & 3 & M & $S$ & Tet & Shooter-E., 2008 \\
\hline Bicolor Beautiful & 3 & M & $\mathrm{D}$ & Tet & Carpenter-J., 2006 \\
\hline Big Navy & 2 & EM & $\mathrm{E}$ & Tet & Stamile, 2006 \\
\hline Big Ross & 1.5 & MLa & $S$ & Dip & Bachman, 2001 \\
\hline Big Yellow Taxi & 3 & EM & $\mathrm{E}$ & Tet & Trimmer, 2005 \\
\hline Bill Chapman & 3 & M & $\mathrm{E}$ & Tet & Kirchhoff-D., 2005 \\
\hline Bit of Blue & 2 & $\mathrm{EE}$ & $\mathrm{E}$ & Tet & Stamile, 2004 \\
\hline Black Fathom Depths & 2.5 & EM & $\mathrm{E}$ & Tet & Salter-E.H., 2008 \\
\hline Blink of an Eye & 3 & $\mathrm{E}$ & S & Dip & Stamile-G., 2004 \\
\hline Blissful Blackberry Pie & 2 & M & $S$ & Tet & Salter, 2005 \\
\hline Blue Beat & 2 & M & $\mathrm{E}$ & Tet & Stamile, 2008 \\
\hline Blue Elf & 2 & EM & $S$ & Dip & Stamile-G., 2006 \\
\hline Blue Flirt & 2 & EM & $S$ & Dip & Stamile-G., 2001 \\
\hline Blue Lake & 3 & $\mathrm{E}$ & $\mathrm{E}$ & Tet & Stamile, 2007 \\
\hline Blue Mountains & 3 & M & $\mathrm{E}$ & Tet & Stamile, 2007 \\
\hline Blue Ribbon Roundup & 2 & M & $\mathrm{E}$ & Dip & Payne-L.H., 2006 \\
\hline Bluebird Butterfly & 1 & MLa & $\mathrm{E}$ & Tet & Stamile, 2007 \\
\hline Bluegrass Music & 3 & EM & $S$ & Tet & Grace-Smith, 2005 \\
\hline Blues Music & 2 & M & $S$ & Tet & Salter, 2005 \\
\hline Bodacious Charmer & 2 & EM & $\mathrm{E}$ & Tet & Carpenter-J., 2007 \\
\hline
\end{tabular}


Table 1. (Continued) Daylily rust severity ratings on 575 daylily cultivars growing in a large landscape planting in southern Mississippi in Aug. 2013. Plants had not received any fungicide treatment during the 2013 growing season. Weather conditions during the growing season were favorable for daylily rust.

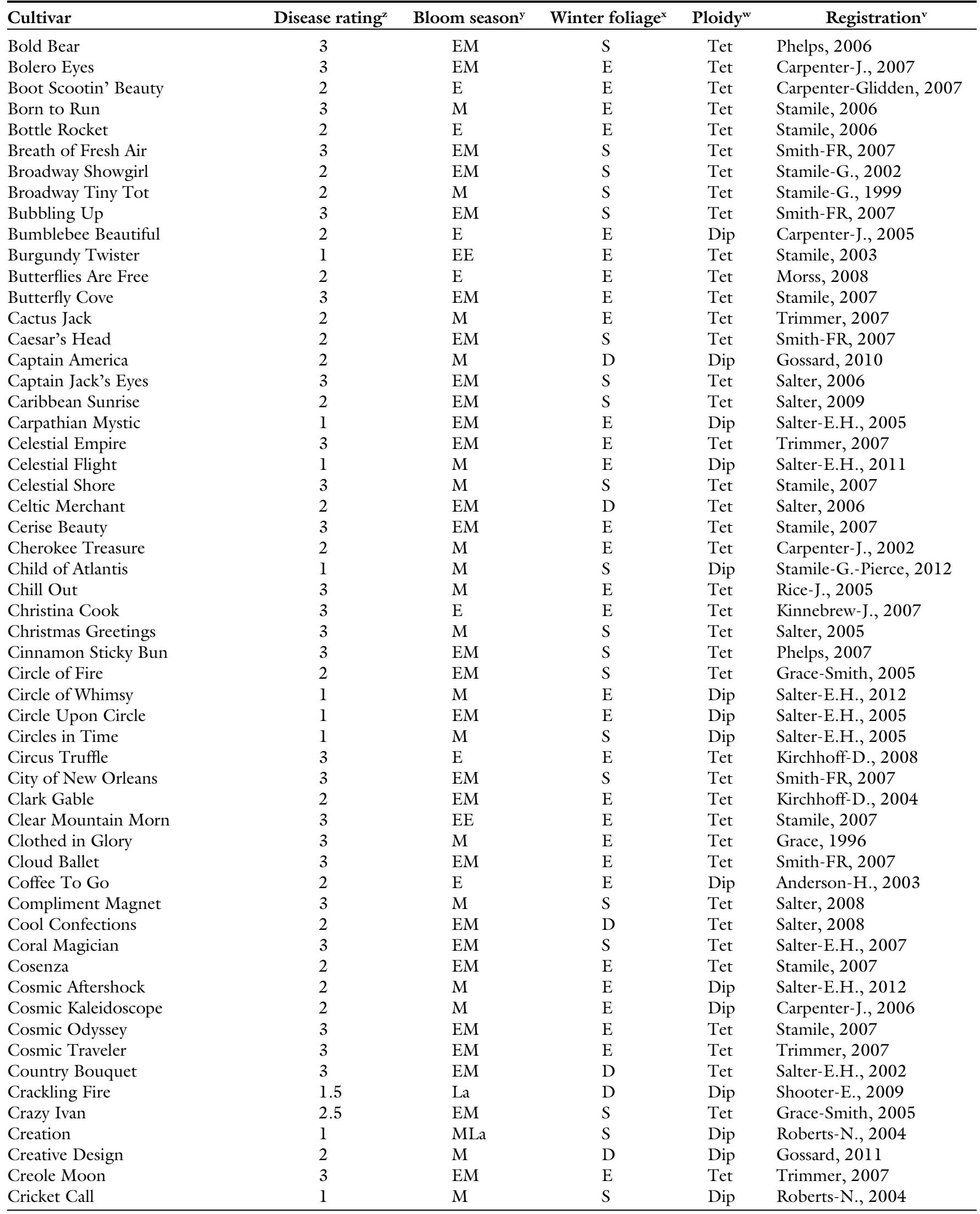

(Continued on next page) 
Table 1. (Continued) Daylily rust severity ratings on 575 daylily cultivars growing in a large landscape planting in southern Mississippi in Aug. 2013. Plants had not received any fungicide treatment during the 2013 growing season. Weather conditions during the growing season were favorable for daylily rust.

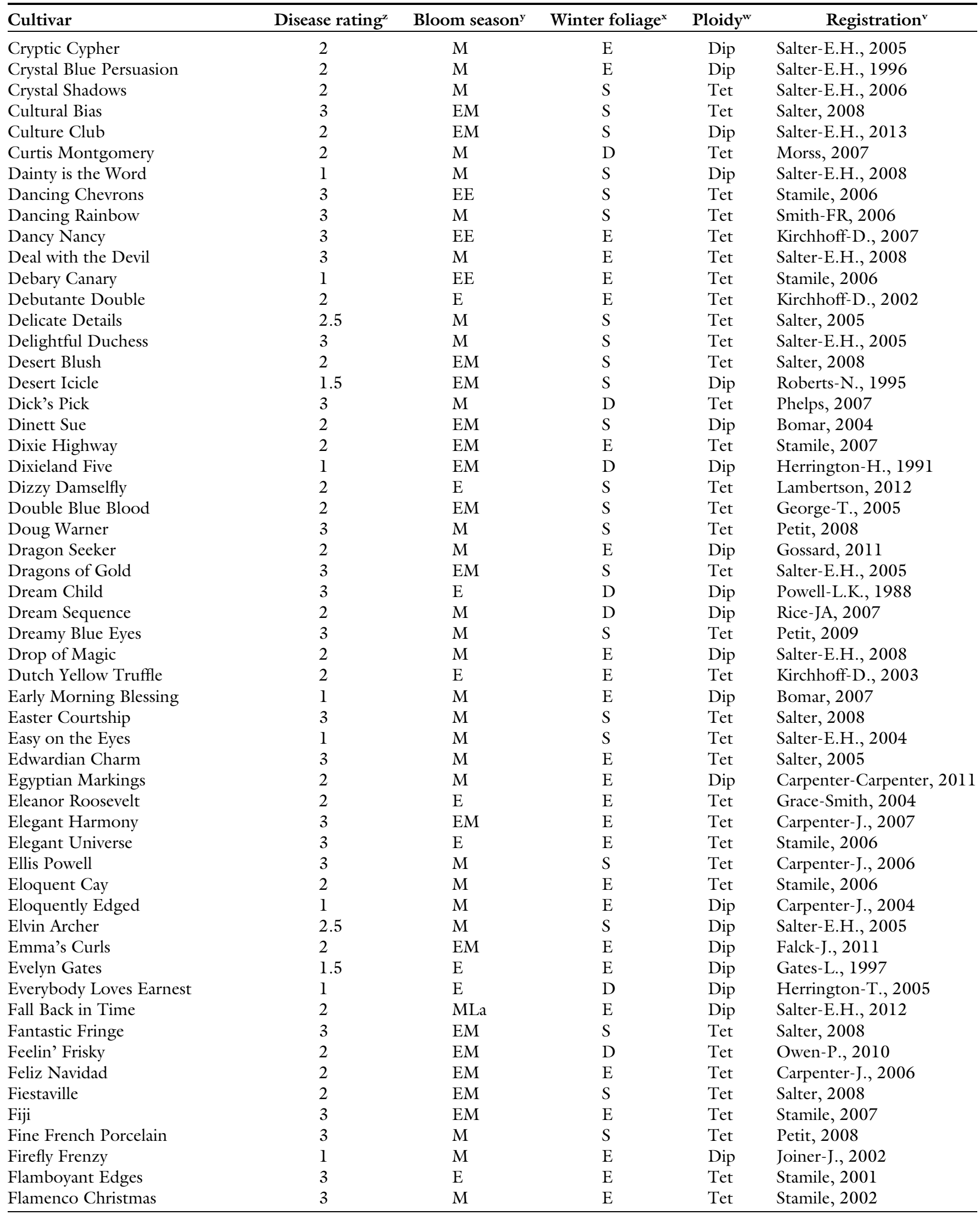

(Continued on next page) 
Table 1. (Continued) Daylily rust severity ratings on 575 daylily cultivars growing in a large landscape planting in southern Mississippi in Aug. 2013. Plants had not received any fungicide treatment during the 2013 growing season. Weather conditions during the growing season were favorable for daylily rust.

\begin{tabular}{|c|c|c|c|c|c|}
\hline Cultivar & Disease rating $^{\mathrm{z}}$ & Bloom season $^{\mathrm{y}}$ & Winter foliage $^{\mathrm{x}}$ & Ploidy $^{\mathrm{w}}$ & Registration $^{\mathrm{v}}$ \\
\hline Flames of Beauty & 2 & $\mathrm{E}$ & $S$ & Tet & Salter-E.H., 2008 \\
\hline Flavored Peach & 3 & $\mathrm{E}$ & $S$ & Tet & Salter, 2008 \\
\hline Flower Faces & 2 & MLa & $\mathrm{E}$ & Dip & Salter-E.H., 2008 \\
\hline Flying Monkeys & 2 & EM & $\mathrm{D}$ & Dip & Herrington-K., 2008 \\
\hline Frank's Adorable Candy & 3 & EM & $\mathrm{E}$ & Tet & Smith-FR, 2007 \\
\hline Frank's Black Eyed Susan & 3 & EM & $S$ & Tet & Smith-FR, 2009 \\
\hline Frilly Bliss & 1 & MLa & $\mathrm{E}$ & Dip & Joiner-J., 1998 \\
\hline Gail Reese & 3 & M & $\mathrm{E}$ & Dip & Reese-R., 2001 \\
\hline Gale's Glory & 3 & EM & $S$ & Tet & Phelps, 2006 \\
\hline Garden Butterfly & 1 & M & $\mathrm{D}$ & Dip & Gossard, 2011 \\
\hline Gary Colby & 2 & M & $S$ & Tet & Petit, 2005 \\
\hline Geometric Wizard & 2 & EM & $S$ & Tet & Salter-E.H., 2012 \\
\hline God Save the Queen & 2.5 & $\mathrm{E}$ & $\mathrm{E}$ & Tet & Morss, 2005 \\
\hline Godsend & 2 & M & $\mathrm{E}$ & Tet & Jaques, 2012 \\
\hline Going Green & 3 & EM & $\mathrm{E}$ & Tet & Stamile, 2007 \\
\hline Goldenzelle & 2 & M & $S$ & Tet & Smith-FR, 2006 \\
\hline Googies & 2 & M & $S$ & Tet & Rice-J., 2005 \\
\hline Gorgeous Smile & 2 & MLa & $\mathrm{D}$ & Tet & Rice-Potter, 2001 \\
\hline Got the Blues & 1 & EM & $S$ & Dip & Stamile-G., 2002 \\
\hline Grapefruit Truffle & 2 & $\mathrm{La}$ & $\mathrm{E}$ & Tet & Kirchhoff-D., 2002 \\
\hline Green Rainbow & 1 & EM & $\mathrm{D}$ & Dip & Herrington-T., 2005 \\
\hline Green Revolution & 3 & EM & $\mathrm{E}$ & Tet & Stamile, 2007 \\
\hline Green Treat & 1.5 & $M$ & $S$ & Dip & Salter-E.H., 2002 \\
\hline Groovy Chick & 3 & EM & $S$ & Tet & Pickles, 2007 \\
\hline Heaven's Majesty & 2 & $\mathrm{E}$ & $S$ & Tet & Carpenter-J., 2006 \\
\hline Heavens Rest & 2 & EM & $\mathrm{E}$ & Tet & Salter-E.H., 2005 \\
\hline Helix & 2 & $\mathrm{EE}$ & $\mathrm{E}$ & Tet & Stamile, 2002 \\
\hline His Highness & 3 & $\mathrm{EE}$ & $\mathrm{E}$ & Tet & Stamile, 2000 \\
\hline Hog Heaven & 1 & $\mathrm{EM}$ & $S$ & Tet & Smith-FR, 2007 \\
\hline Hold Your Horses & 2.5 & EM & $\mathrm{E}$ & Tet & Trimmer, 2004 \\
\hline Holy Water & 3 & EM & $S$ & Tet & Smith-FR, 2007 \\
\hline Home Run King & 1.5 & $\mathrm{E}$ & $\mathrm{E}$ & Dip & Carpenter-J., 2009 \\
\hline Hot and Hip & 2 & EM & $S$ & Tet & Salter-E.H., 2012 \\
\hline Hot Flashes & 3 & EM & $S$ & Tet & Phelps, 2007 \\
\hline Hot Scheme & 2 & M & $S$ & Tet & Salter-E.H., 1997 \\
\hline How Lovely You Are & 2 & MLa & $S$ & Tet & Rice-J., 2006 \\
\hline Hunters Quest & 1 & $M$ & $\mathrm{D}$ & Tet & Taylor-O., 1981 \\
\hline I Know Who Holds Tomorrow & 2 & M & $\mathrm{D}$ & Dip & Bomar, 2005 \\
\hline I Remember You & 2 & EM & $S$ & Tet & Smith-FR, 2005 \\
\hline Ida Mae Norris & 2 & M & $\mathrm{D}$ & Dip & Norris-R., 2009 \\
\hline Irish Icon & 1 & M & $\mathrm{E}$ & Dip & Salter-E.H., 2008 \\
\hline Isabelle Phelps & 3 & M & $S$ & Dip & Phelps, 2006 \\
\hline Isabelle's Inspiration & 2.5 & M & $S$ & Tet & Phelps, 2006 \\
\hline Isle of Wight & 2 & $\mathrm{E}$ & $\mathrm{E}$ & Tet & Trimmer, 2004 \\
\hline
\end{tabular}

(Continued on next page) 
Table 1. (Continued) Daylily rust severity ratings on 575 daylily cultivars growing in a large landscape planting in southern Mississippi in Aug. 2013. Plants had not received any fungicide treatment during the 2013 growing season. Weather conditions during the growing season were favorable for daylily rust.

\begin{tabular}{|c|c|c|c|c|c|}
\hline Cultivar & Disease rating ${ }^{\mathrm{z}}$ & Bloom season ${ }^{\mathrm{y}}$ & Winter foliage ${ }^{\mathrm{x}}$ & Ploidy $^{w}$ & Registration $^{\mathrm{v}}$ \\
\hline It Is Finished & 3 & EM & $\mathrm{E}$ & Tet & Carpenter-J., 2004 \\
\hline J.J. Homsey & 2 & M & $\mathrm{E}$ & Tet & Homsey-P, 2004 \\
\hline J.T. Davis & 1 & M & $\mathrm{E}$ & Tet & Grace-L., 1999 \\
\hline Jacki Kropf & 2 & EM & $S$ & Tet & Morss, 2004 \\
\hline Jade Glow & 3 & EM & $\mathrm{E}$ & Tet & Stamile, 2005 \\
\hline James Fennell & 2 & M & $\mathrm{E}$ & Dip & Salter-E.H., 2013 \\
\hline Jealous Dancer & 3 & $\mathrm{E}$ & $\mathrm{D}$ & Dip & Carpenter-J., 2007 \\
\hline Jennifer Trimmer & 3 & EM & $\mathrm{E}$ & Tet & Trimmer, 2006 \\
\hline Johnny Bear & 2 & $\mathrm{EE}$ & $\mathrm{E}$ & Tet & Hansen-D., 2009 \\
\hline Jolly Red Giant & 3 & $\mathrm{E}$ & $\mathrm{E}$ & Tet & Stamile, 1999 \\
\hline Journey to $\mathrm{Oz}$ & 2 & EM & $\mathrm{D}$ & Dip & Herrington-K., 2001 \\
\hline Joy of Life & 1.5 & M & $\mathrm{E}$ & Tet & Carpenter-J., 2006 \\
\hline Just for Breakfast & 2 & EM & $S$ & Dip & Threewitts, 1994 \\
\hline Kaleidoscope Jungle Cat & 1 & M & $\mathrm{E}$ & Dip & Carpenter-Carpenter, 2011 \\
\hline Kaleidoscope Puzzle & 1.5 & EM & $\mathrm{E}$ & Dip & Carpenter-Carpenter, 2010 \\
\hline Kaleidoscopic Intrigue & 1 & M & $\mathrm{E}$ & Dip & Carpenter-J., 2009 \\
\hline Katisue & 2 & MLa & $\mathrm{D}$ & Dip & Lewis-J., 1989 \\
\hline Kings Point & 2 & M & $S$ & Tet & Trimmer, 2004 \\
\hline Kiss My Grits & 2 & M & $S$ & Tet & Smith-FR, 2007 \\
\hline Korean Kite & 2 & M & $S$ & Tet & Salter-E.H., 2005 \\
\hline Lacy Border & 2 & $\mathrm{E}$ & $\mathrm{E}$ & Tet & Stamile, 2006 \\
\hline Lacy Dusk & 2 & EE & $S$ & Tet & Stamile, 2007 \\
\hline Lady Betty Fretz & 3 & M & $S$ & Tet & Petit, 2005 \\
\hline Lala Fabulosa & 3 & M & $S$ & Tet & Smith-FR, 2007 \\
\hline Leonardo's Perspective of Color & 1 & M & $S$ & Dip & Faulkner, 2011 \\
\hline Let Loose & 2 & EE & $\mathrm{E}$ & Tet & Stamile, 2003 \\
\hline Levi Davis & 3 & M & $\mathrm{E}$ & Tet & Carpenter-J., 2005 \\
\hline Light and Lively & 2 & EM & $S$ & Tet & Salter, 2008 \\
\hline Lillian's Jack Temple & 1 & M & $S$ & Dip & Manning, 2007 \\
\hline Lillian’s Jinger Bred & 2 & M & $\mathrm{E}$ & Tet & Manning, 2007 \\
\hline Lillian's Woman's Touch & 2 & M & $\mathrm{E}$ & Dip & Manning, 2002 \\
\hline Linda Beck & 2 & M & $S$ & Tet & Agin, 2005 \\
\hline Linda's Magic & 3 & EM & $S$ & Tet & Grace-Smith, 2003 \\
\hline Lisa's Smile & 3 & M & $S$ & Tet & Phelps, 2006 \\
\hline Listen Missy & 2 & M & $S$ & Tet & Phelps, 2006 \\
\hline Little Blue Belle & 1 & EM & $S$ & Dip & Stamile-G., 2006 \\
\hline Little Enchantress & 2 & $\mathrm{E}$ & $\mathrm{D}$ & Dip & Stamile-G., 2004 \\
\hline Little Gold Nugget & 1.5 & EM & $\mathrm{D}$ & Dip & Herrington-T., 2005 \\
\hline Little Gypsy Girl & 2 & $\mathrm{E}$ & $S$ & Dip & Stamile-G., 2003 \\
\hline Little Pink Cloud & 1 & $\mathrm{E}$ & $S$ & Dip & Winniford-E., 1985 \\
\hline Little Raven & 1 & EM & $S$ & Dip & Stamile-G., 2006 \\
\hline Little Red Dumples & 1 & $\mathrm{E}$ & $\mathrm{D}$ & Dip & Anderson-H., 2000 \\
\hline Little Secrets & 2 & EM & $S$ & Dip & Stamile-G., 2006 \\
\hline Little Trooper & 1 & EM & $S$ & Dip & Stamile-G., 2008 \\
\hline
\end{tabular}


Table 1. (Continued) Daylily rust severity ratings on 575 daylily cultivars growing in a large landscape planting in southern Mississippi in Aug. 2013. Plants had not received any fungicide treatment during the 2013 growing season. Weather conditions during the growing season were favorable for daylily rust.

\begin{tabular}{|c|c|c|c|c|c|}
\hline Cultivar & Disease rating $^{\mathrm{z}}$ & Bloom season $^{\mathrm{y}}$ & Winter foliage $\mathrm{e}^{\mathrm{x}}$ & Ploidy $^{\mathrm{w}}$ & Registration $^{\mathrm{v}}$ \\
\hline Little Wizard & 1 & M & $S$ & Dip & Stamile-G., 2008 \\
\hline Lone Star Wagon Wheel & 2 & EM & $S$ & Dip & Payne-L.H., 2004 \\
\hline Lonesome Heart & 3 & M & $\mathrm{E}$ & Tet & Stamile, 2006 \\
\hline Long Tall Sally & 2 & EM & $\mathrm{D}$ & Tet & Trimmer, 1996 \\
\hline Look Here Mary & 1.5 & M & $S$ & Dip & Cranshaw, 1986 \\
\hline Lost in Illusion & 2 & M & $\mathrm{E}$ & Dip & Salter-E.H., 2011 \\
\hline Magic Maker & 2 & M & $S$ & Dip & Gossard, 2011 \\
\hline Magic of $\mathrm{Oz}$ & 2 & M & $\mathrm{D}$ & Dip & Herrington-H., 1995 \\
\hline Magical Mystery Show & 3 & $\mathrm{E}$ & $\mathrm{E}$ & Tet & Stamile, 2007 \\
\hline Magnificent Obsession & 3 & EM & $S$ & Tet & Smith-FR, 2007 \\
\hline Make Your Point & 1.5 & M & $\mathrm{E}$ & Dip & Salter-E.H., 2008 \\
\hline Malaysian Marketplace & 3 & EM & $S$ & Tet & Salter, 2005 \\
\hline Mars Everlasting Fury & 2 & EM & $\mathrm{E}$ & Dip & Salter-A., 2009 \\
\hline Marseilles Watercolor & 1 & $\mathrm{EE}$ & $\mathrm{E}$ & Tet & Stamile, 2007 \\
\hline Mary Alice & 3 & EM & $S$ & Tet & Smith-FR, 2007 \\
\hline Mary Ethel Anderson & 2 & MLa & $S$ & Dip & Salter-E.H., 1995 \\
\hline Masked Marauder & 3 & M & $S$ & Tet & Petit, 2010 \\
\hline Maude Reese & 1 & $\mathrm{E}$ & $\mathrm{E}$ & Dip & Reese-R., 2008 \\
\hline McKenzie Agin & 3 & M & $\mathrm{D}$ & Tet & Agin, 2007 \\
\hline Medicine Feather & 2 & EM & $S$ & Dip & Roberts-N., 2001 \\
\hline Mediterranean Muse & 2 & M & $S$ & Tet & Salter, 2006 \\
\hline Memories Remain & 2 & EM & $\mathrm{E}$ & Tet & Carr, 2005 \\
\hline Merely Mystical & 3 & $\mathrm{EE}$ & $S$ & Tet & Salter-E.H., 2008 \\
\hline Merlin's Prophecy & 1 & $M$ & $S$ & Dip & Salter-E.H., 2008 \\
\hline Mitch Ensor & 3 & EM & $S$ & Tet & Phelps, 2007 \\
\hline Mix and Match & 1 & M & $\mathrm{D}$ & Tet & Salter-E.H., 2012 \\
\hline Mixed Berry Jam & 2 & M & $S$ & Dip & Salter-E.H., 2009 \\
\hline Moon Magic & 1 & M & $\mathrm{D}$ & Dip & Womble, 1949 \\
\hline Moon Sworn & 2 & $M$ & $\mathrm{E}$ & Dip & Salter-E.H., 2013 \\
\hline Morning All Day & 1 & EM & $\mathrm{D}$ & Dip & Herrington-K., 2001 \\
\hline Morrie Otte & 2 & $M$ & $S$ & Dip & Salter-E.H., 1996 \\
\hline Mount Herman Morning Beauty & 3 & M & $\mathrm{E}$ & Tet & Carpenter-J., 2007 \\
\hline Much Ado About Magic & 3 & M & $S$ & Tet & Salter, 2005 \\
\hline My Friend Floyd & 1 & M & $S$ & Dip & Roberts-N., 2005 \\
\hline My Sunny Valentine & 3 & M & $S$ & Tet & Salter, 2007 \\
\hline Natural Born Charmer & 2 & EM & $S$ & Tet & Salter-E.H., 2007 \\
\hline Navajo Princess & 2 & MLa & $S$ & Dip & Hansen, 1992 \\
\hline Nebula on Fire & 2 & EM & $S$ & Tet & Grace-Smith, 2005 \\
\hline New Hampshire Heaven & 3 & $M$ & $\mathrm{E}$ & Tet & Carpenter-J., 2006 \\
\hline Nile Jewel & 2 & M & $\mathrm{E}$ & Dip & Salter-E.H., 2006 \\
\hline Off To See the Wizard & 2 & M & $S$ & Dip & Herrington-K., 2009 \\
\hline Oh So Awesome & 3 & M & S & Tet & Salter, 2008 \\
\hline On the Lookout & 2 & M & $\mathrm{E}$ & Tet & Salter-E.H., 2005 \\
\hline One Way Home & 2 & EM & $S$ & Tet & Smith-FR, 2007 \\
\hline
\end{tabular}

(Continued on next page) 
Table 1. (Continued) Daylily rust severity ratings on 575 daylily cultivars growing in a large landscape planting in southern Mississippi in Aug. 2013. Plants had not received any fungicide treatment during the 2013 growing season. Weather conditions during the growing season were favorable for daylily rust.

\begin{tabular}{|c|c|c|c|c|c|}
\hline Cultivar & Disease rating $^{\mathrm{z}}$ & Bloom season $^{\mathrm{y}}$ & Winter foliage ${ }^{\mathrm{x}}$ & Ploidy $^{w}$ & Registration $^{\mathrm{v}}$ \\
\hline Only Believe & 3 & EM & $S$ & Tet & Grace-L., 1998 \\
\hline Our Friend Tom Wilson & 1 & EM & $\mathrm{E}$ & Dip & Carpenter-J., 2004 \\
\hline Out in Style & 3 & M & $S$ & Tet & Salter-E.H., 1996 \\
\hline Out of the Blue & 1 & M & $\mathrm{E}$ & Dip & Salter-E.H., 2002 \\
\hline Over the Line & 1 & M & $\mathrm{E}$ & Dip & Salter-E.H., 2005 \\
\hline Over the Mountain & 3 & M & $\mathrm{E}$ & Tet & Stamile, 2007 \\
\hline Parting the Red Sea & 2 & EE & $\mathrm{E}$ & Tet & Hansen-D., 2009 \\
\hline Passion’s Blush & 3 & $\mathrm{E}$ & $S$ & Tet & Phelps, 2007 \\
\hline Pathway to Paradise & 3 & M & $\mathrm{E}$ & Tet & Salter, 2005 \\
\hline Pattern Pleaser & 1 & $\mathrm{E}$ & $\mathrm{E}$ & Dip & Carpenter-J., 2009 \\
\hline Peach Cupcake & 1 & EM & $\mathrm{D}$ & Dip & Herrington-T., 2000 \\
\hline Peach Wine & 3 & EM & $S$ & Tet & Phelps, 2006 \\
\hline Pigment of Imagination & 2 & M & $\mathrm{D}$ & Dip & Norris-R., 2008 \\
\hline Pineapple Moon & 2 & EM & $\mathrm{E}$ & Tet & Trimmer, 2007 \\
\hline Pink Enchilada & 1 & $M$ & $\mathrm{D}$ & Dip & Roberts-N., 2005 \\
\hline Pink Lemonade & 3 & EM & $\mathrm{E}$ & Dip & Durio, 1975 \\
\hline Pink Lemonade Party & 2 & M & $S$ & Tet & Salter, 2006 \\
\hline Pinwheel Princess & 1 & EM & $\mathrm{E}$ & Dip & Salter-E.H., 2010 \\
\hline Pirate King & 2.5 & EM & $S$ & Tet & Grace-Smith, 2004 \\
\hline Pixie Pageantry & 3 & MLa & $S$ & Tet & Salter-E.H., 2004 \\
\hline Pixie Pinwheel Party & 1 & M & $\mathrm{E}$ & Dip & Salter-E.H., 2008 \\
\hline Planet Max & 1.5 & EM & $S$ & Dip & Reed, 1996 \\
\hline Pleasantly Plum & 2 & M & $S$ & Tet & Salter, 2008 \\
\hline Plum Cupcake & 1 & EM & $S$ & Dip & Herrington-T., 1998 \\
\hline Pumpkin Mime & 1 & EM & $S$ & Dip & Salter-E.H., 2005 \\
\hline Pure Indulgence & 3 & $\mathrm{E}$ & $\mathrm{E}$ & Tet & Carr, 2000 \\
\hline Purple Suspenders & 1 & M & $\mathrm{D}$ & Tet & Webster-Cobb, 1999 \\
\hline Quartz Rainbow & 2 & EE & $\mathrm{E}$ & Tet & Stamile, 2007 \\
\hline Quiet Reflections & 2 & EM & $\mathrm{E}$ & Tet & Salter, 2006 \\
\hline Radioactive Curls & 2 & M & $\mathrm{E}$ & Dip & Gossard, 2004 \\
\hline Random Thoughts & 2 & EM & $\mathrm{E}$ & Tet & Salter-E.H., 2005 \\
\hline Raven's Rage & 3 & M & $S$ & Tet & Salter-E.H., 2002 \\
\hline Razor Eyes & 2 & M & $S$ & Tet & Lambertson, 2006 \\
\hline Red Done Right & 3 & EM & $S$ & Tet & Salter, 2008 \\
\hline Red Handlebars & 1 & MLa & $\mathrm{D}$ & Dip & Roberts-N., 2003 \\
\hline Refuge Point & 2 & EM & $S$ & Tet & Salter-E.H., 2008 \\
\hline Regal Fantasy & 2 & EM & $S$ & Tet & Salter, 2005 \\
\hline Reggie Morgan & 3 & M & $\mathrm{D}$ & Tet & Petit, 2008 \\
\hline Return to $\mathrm{Oz}$ & 2 & M & $\mathrm{D}$ & Dip & Herrington-K., 2000 \\
\hline Rhubarb Pie & 2 & $\mathrm{E}$ & $\mathrm{E}$ & Tet & Stamile, 2007 \\
\hline Ring the Bells of Heaven & 3 & EM & $\mathrm{D}$ & Tet & Carpenter-J., 2006 \\
\hline Rita Sue & 3 & M & $S$ & Tet & Smith-FR, 2006 \\
\hline Road to Dixie & 3 & EM & $S$ & Tet & Salter-E.H., 2005 \\
\hline Robert W. Grenkowitz & 3 & M & $S$ & Tet & Petit, 2008 \\
\hline
\end{tabular}

(Continued on next page) 
Table 1. (Continued) Daylily rust severity ratings on 575 daylily cultivars growing in a large landscape planting in southern Mississippi in Aug. 2013. Plants had not received any fungicide treatment during the 2013 growing season. Weather conditions during the growing season were favorable for daylily rust.

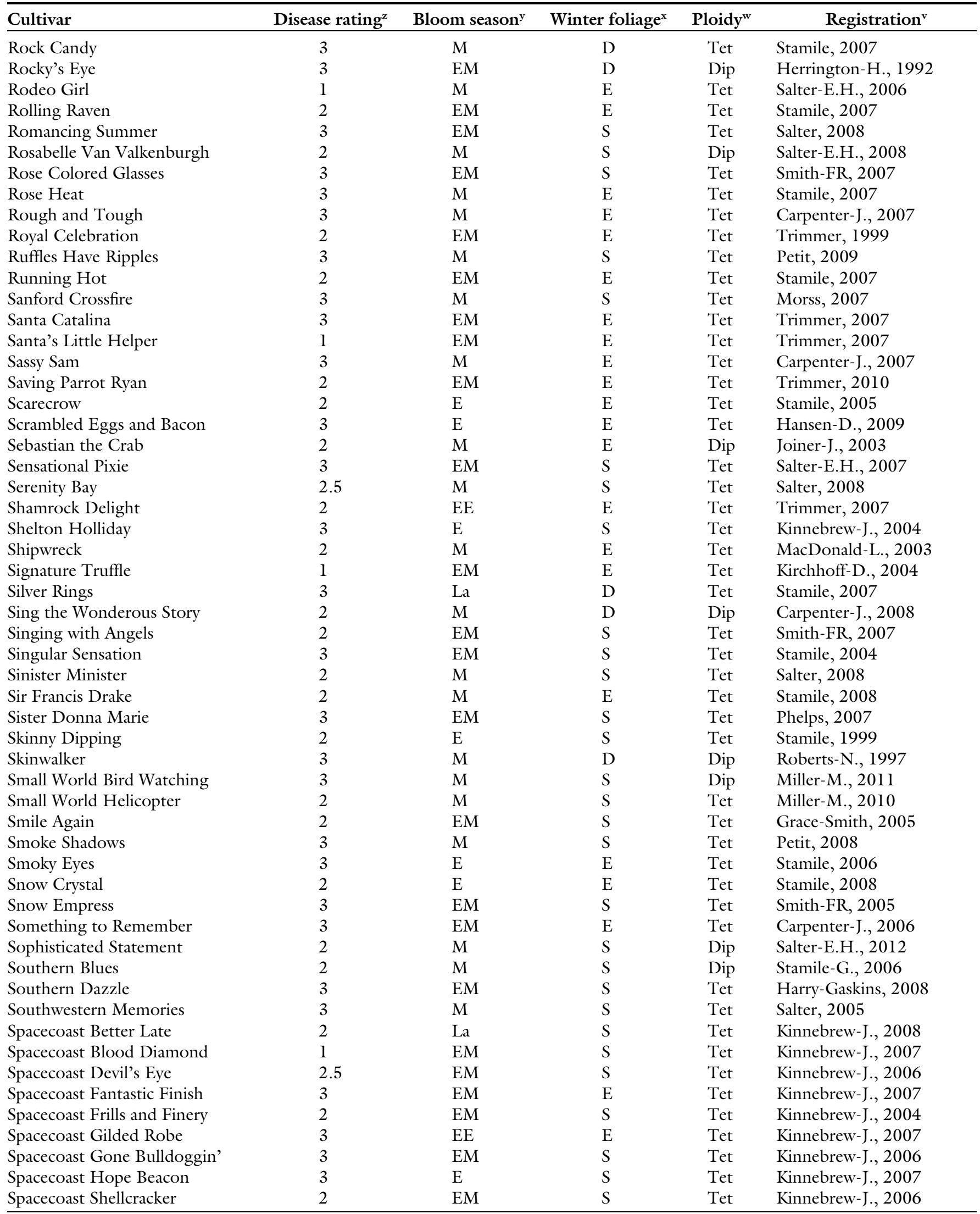

(Continued on next page) 
Table 1. (Continued) Daylily rust severity ratings on 575 daylily cultivars growing in a large landscape planting in southern Mississippi in Aug. 2013. Plants had not received any fungicide treatment during the 2013 growing season. Weather conditions during the growing season were favorable for daylily rust.

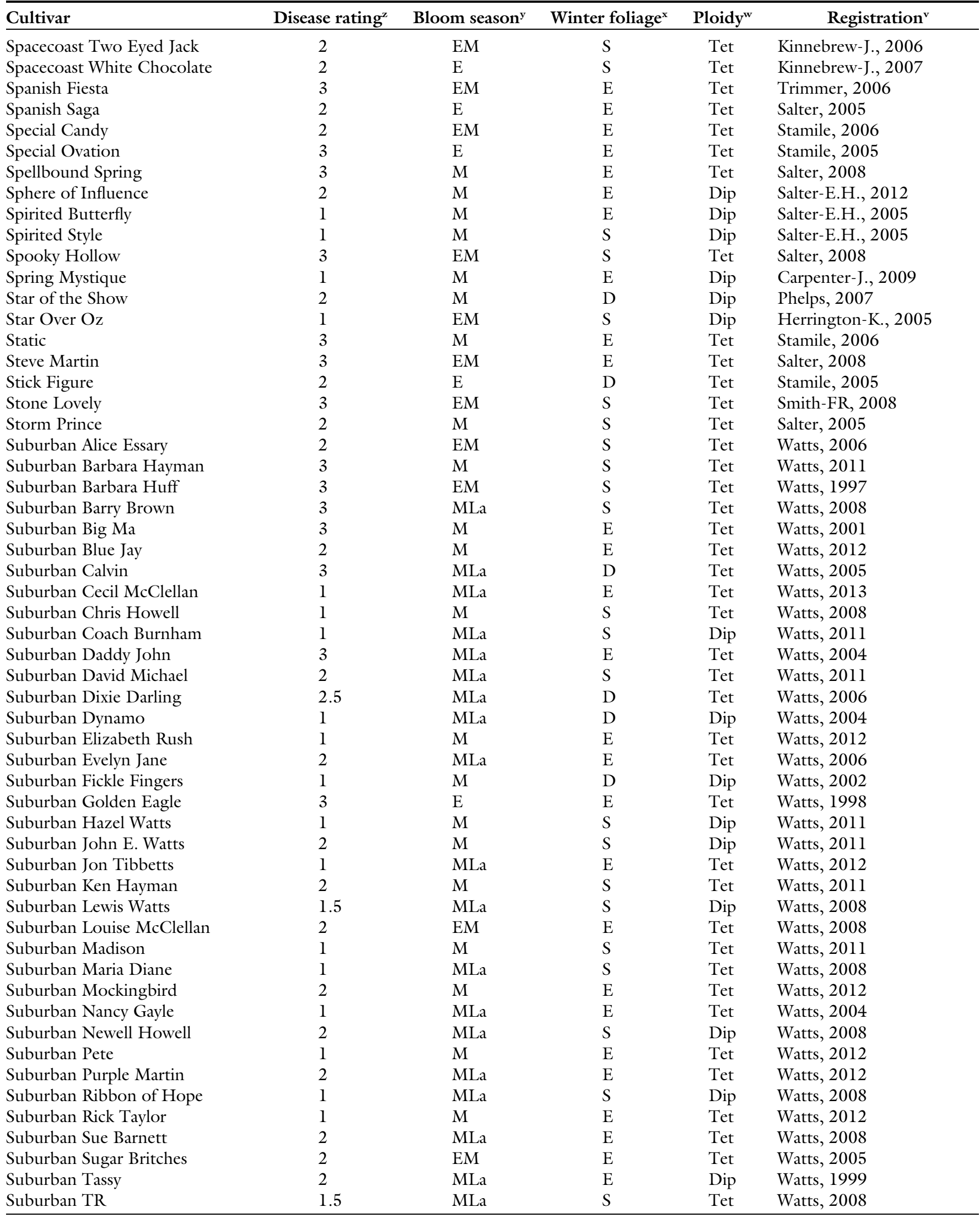


Table 1. (Continued) Daylily rust severity ratings on $\mathbf{5 7 5}$ daylily cultivars growing in a large landscape planting in southern Mississippi in Aug. 2013. Plants had not received any fungicide treatment during the 2013 growing season. Weather conditions during the growing season were favorable for daylily rust.

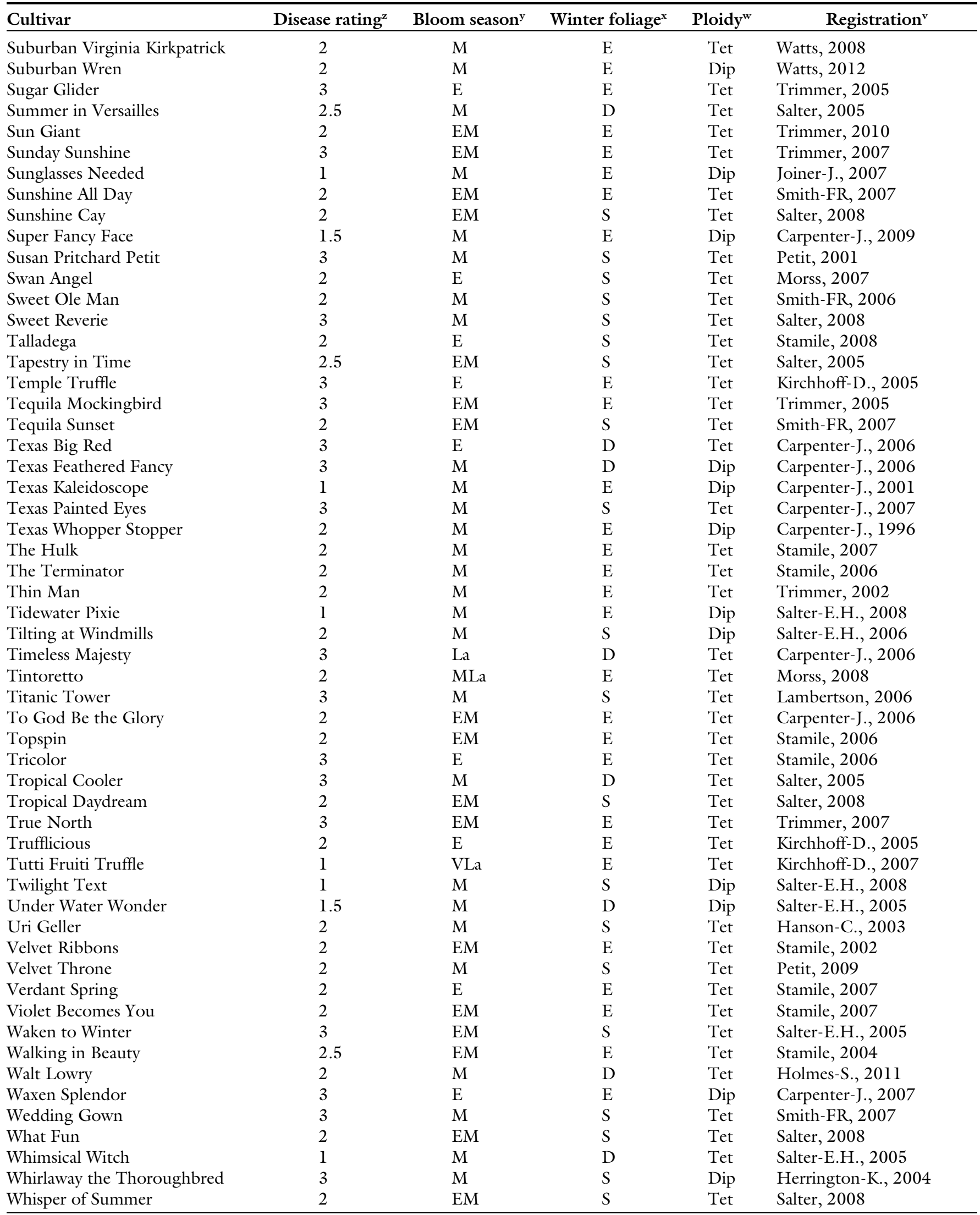


Table 1. (Continued) Daylily rust severity ratings on 575 daylily cultivars growing in a large landscape planting in southern Mississippi in Aug. 2013. Plants had not received any fungicide treatment during the 2013 growing season. Weather conditions during the growing season were favorable for daylily rust.

\begin{tabular}{|c|c|c|c|c|c|}
\hline Cultivar & Disease rating $^{\mathrm{z}}$ & Bloom season $^{\mathrm{y}}$ & Winter foliage $^{\mathrm{x}}$ & Ploidy ${ }^{w}$ & Registration $^{\mathrm{v}}$ \\
\hline White on Rice & 2 & EM & $S$ & Tet & Smith-FR, 2009 \\
\hline Who Do Voodoo & 3 & EM & $\mathrm{E}$ & Tet & Kinnebrew-J., 2007 \\
\hline Wild and Free & 1 & $\mathrm{E}$ & $\mathrm{E}$ & Tet & Stamile, 2005 \\
\hline Wild Red Heart & 3 & EM & $S$ & Tet & Salter, 2008 \\
\hline Wild Winter Wine & 3 & EM & $\mathrm{E}$ & Tet & Kirchhoff-D., 2007 \\
\hline Wild Wookie & 2 & M & $\mathrm{E}$ & Tet & Stamile, 2002 \\
\hline Windstar & 1 & EM & $\mathrm{D}$ & Dip & Herrington-K., 2001 \\
\hline Wings of Angels & 3 & EM & $S$ & Tet & Grace-Smith, 2005 \\
\hline Winter's Crown & 2 & M & $\mathrm{E}$ & Tet & Salter, 2008 \\
\hline Wizard at Large & 2 & EM & $S$ & Tet & Salter, 2008 \\
\hline Wonder of It All & 3 & $\mathrm{E}$ & $\mathrm{D}$ & Tet & Carr, 2005 \\
\hline World Traveler & 3 & $\mathrm{E}$ & $\mathrm{E}$ & Tet & Trimmer, 2006 \\
\hline
\end{tabular}

${ }^{\mathrm{z}} \mathrm{l}=$ no or little visual sign of infections; 2 = moderate infection; $3=$ severe infection. Most cultivars $(85 \%)$ were represented by a single clump of plants, $12 \%$ by two clumps, and

$3 \%$ by three or more clumps. When there were two or more clumps of the same cultivar, the median is reported as the rating.

${ }^{y} \mathrm{EE}=$ extra early; $\mathrm{E}=$ early; $\mathrm{EM}=$ early midseason; $\mathrm{M}=$ midseason; $\mathrm{MLa}=$ late midseason; La = late; VLa = very late (American Hemerocallis Society, 2015).

${ }^{\mathrm{D}} \mathrm{D}=$ dormant $\mathrm{S}=$ semievergreen; $\mathrm{E}=$ evergreen (American Hemerocallis Society, 2015)

${ }^{\mathrm{w}}$ Dip = diploid; Tet $=$ tetraploid (American Hemerocallis Society, 2015).

'Originator of the cultivar and year of registration with the American Hemerocallis Society (2015).

Table 2. Mean maximum, average, and minimum daily air temperature; mean daily dew point, and total precipitation in June, July, and Aug. 2013 in Hattiesburg, MS. ${ }^{\mathrm{z}}$

\begin{tabular}{lccccc}
\hline Month & Maximum temp $\left({ }^{\circ} \mathbf{F}\right)^{\mathrm{y}}$ & $\operatorname{Avg}$ temp $\left({ }^{\circ} \mathbf{F}\right)$ & Minimum temp $\left({ }^{\circ} \mathbf{F}\right)$ & Dew point $\left({ }^{\circ} \mathbf{F}\right)$ & Precipitation $(\text { inches })^{\mathrm{y}}$ \\
\hline June & 91 & 81 & 70 & 71 & 5.08 \\
July & 89 & 80 & 71 & 72 & 5.85 \\
August & 90 & 81 & 71 & 72 & 4.03 \\
\hline
\end{tabular}

${ }^{2}$ Weather history for Hattiesburg Bobby L. Chain Municipal Airport, Hattiesburg, MS (Weather Underground, 2013).

${ }^{\circ}\left({ }^{\circ}-32\right) \div 1.8={ }^{\circ} \mathrm{C}, 1$ inch $=2.54 \mathrm{~cm}$

Identification of rust-resistant and rust-susceptible daylily cultivars is of benefit to breeders, commercial growers, and home gardeners, particularly in helping to reduce the time and expense involved in repeated fungicide applications in nurseries and landscapes. In Georgia, WilliamsWoodward and Buck (2001) conducted a preliminary greenhouse study with pathogen-inoculated plants of 22 cultivars and Mueller et al. (2003) followed with a greenhouse study with pathogen-inoculated plants of 84 cultivars to assess relative resistance to daylily rust. Robbins and Vann (2002) surveyed 500 fieldgrown cultivars at a small commercial nursery in central Arkansas in 2001, with a follow-up survey of 500 cultivars in 2002 (Robbins, 2003), with 75\% of these cultivars also surveyed in the prior year.

As of early 2015 , over 78,000 cultivars have been registered with the American Hemerocallis Society (2015). Little information is available on resistance and susceptibility of daylily cultivars that have been developed and registered since 2000 . This report presents results from a survey of 575 mostly modern (registered from 2000 onward) hybrid cultivars of daylily growing in a landscape in southern Mississippi under conditions favorable for daylily rust.

\section{Materials and methods}

In Aug. 2013, a large, established, residential landscape planting of 575 daylily cultivars (Table $\mathbf{1}$ ) in Hattiesburg, MS, was made available on a short-term basis for a survey of daylily rust severity. Because of planned removal of the planting, the daylily plants had not been treated with any fungicides during 2013 . The 2013 growing season was characterized by warm temperatures, humid conditions, and regular rainfall, with conditions being favorable for daylily rust (Table 2 ). The planting was irrigated daily with overhead sprinklers.
The 575 cultivars in the surveyed planting consisted of mostly modern hybrids, with $93 \%$ of the cultivars registered in 2000 or later and $76 \%$ of the cultivars registered in 2005 or later. Most cultivars (85\%) were represented by a single clump of plants, $12 \%$ by two clumps, and $3 \%$ by three or more clumps. Clumps were well developed and contained a minimum of five fans (ramets), with fan count per clump varying by cultivar. Clumps of the same cultivar were in different locations in the planting. Clumps were spaced on $\approx 3$-ft centers.

Individual clumps of each cultivar were visually assessed for daylily rust on 16 Aug. 2013 and rated by agreement of three raters using an ordinal scale of 1 (no or little sign of infection), 2 (moderate infection), and 3 (severe infection) (Table 1 ). When there were two or more clumps of the same cultivar, the median is reported as the rating. Additional information on each cultivar (bloom season, winter foliage, ploidy, 
originator, and year of registration) was obtained from the database of the American Hemerocallis Society (2015).

For statistical analysis, median rust ratings were grouped into three ordinal categories: 1 and 1.5 (apparently resistant), 2 (apparently moderately susceptible), and 2.5 and 3 (highly susceptible). Ploidy level (diploid or tetraploid) of the cultivars was evaluated for association with the three ratings groups using Fisher's exact test (Freeman-Halton test). Bloom season, as ordinal categories (extra early, early, early midseason, midseason, late midseason, late, and very late), and winter foliage, as ordinal categories (dormant, semievergreen, and evergreen), were evaluated for correlation with the three ordinal ratings groups using the Cochran-Mantel-Haenszel statistic for nonzero correlation and as nominal categories using the Cochran-Mantel-Haenszel statistic for differing row mean scores (analysis of variance-type analysis). For comparison, the same statistics were calculated for cultivars in listings of rust-resistant and rust-susceptible cultivars previously published by Mueller et al. (2003), Robbins and Vann (2002), and Robbins (2003), with cultivar characteristics in the listings supplemented by information obtained from the American Hemerocallis Society (2015) when not provided by the authors. Statistical analyses were conducted using the FREQ procedure of SAS (version 9.4; SAS Institute, Cary, NC).

\section{Results and discussion}

A total of 119 (21\%) of the 575 cultivars in the survey received a rating of 1 or 1.5 and $230(40 \%)$ cultivars received a rating of 2 (Table 1 ), indicating apparent resistance and moderate susceptibility, respectively; however, some of these cultivars may be more susceptible to the disease than a one-time, single-clump rating might reveal. A total of $226(39 \%)$ cultivars received a rating of 2.5 or 3 , indicating them to be highly susceptible to daylily rust, and would likely require frequent applications of fungicides to control the disease. Although most of the daylily cultivars in the survey were represented by only single clumps of plants, the ability to evaluate 575 cultivars in one location in a season with weather conditions favorable for daylily rust, along with the plants having not been treated with fungicides, provided a unique opportunity to collect data on potential disease resistance and susceptibility of these mostly modern hybrid cultivars.

In a preliminary study at the University of Georgia by Williams-
Woodward and Buck (2001) with pathogen-inoculated plants in a greenhouse, 4 cultivars were reported as showing little infection, 9 cultivars being moderately susceptible, and 13 cultivars as being highly susceptible. In further work at the University of Georgia reported by Mueller et al. (2003) with 84 cultivars obtained from three commercial nurseries and

Table 3. Distributions of diploid and tetraploid daylily cultivars among ratings for daylily rust severity in the current survey in a Mississippi landscape in 2013, previous nursery field surveys in Arkansas in 2001 and 2002, and a greenhouse study in Georgia reported in 2003.

\begin{tabular}{|c|c|c|c|c|c|}
\hline \multirow[b]{3}{*}{ Ploidy } & \multicolumn{3}{|c|}{ Cultivars (\% by rating) } & & \multirow[b]{3}{*}{ Cultivars in survey (no.) } \\
\hline & \multicolumn{3}{|c|}{ Mississippi (landscape) ${ }^{\mathrm{z}}$} & & \\
\hline & 1 or 1.5 & 2 & 2.5 or 3 & & \\
\hline Diploid & 51 & 40 & 9 & & 163 \\
\hline \multirow[t]{3}{*}{ Tetraploid } & 9 & $\begin{array}{c}40 \\
P<0.0001^{y}\end{array}$ & 51 & & 412 \\
\hline & \multicolumn{3}{|c|}{ Arkansas (nursery) $2001^{x}$} & & \\
\hline & 1 to 2 & $2<$ rating $<4$ & 4 to 5 & & \\
\hline Diploid & 78 & 18 & 4 & & 293 \\
\hline \multirow[t]{3}{*}{ Tetraploid } & 69 & $\begin{array}{c}28 \\
P=0.0237\end{array}$ & 3 & & 195 \\
\hline & \multicolumn{3}{|c|}{ Arkansas (nursery) $2002^{\mathrm{w}}$} & & \\
\hline & 1 to 2 & $2<$ rating $<4$ & 4 to 5 & & \\
\hline Diploid & 82 & 9 & 9 & & 313 \\
\hline \multirow[t]{3}{*}{ Tetraploid } & 50 & $\begin{array}{c}21 \\
P<0.0001\end{array}$ & 29 & & 187 \\
\hline & \multicolumn{4}{|c|}{ Georgia (greenhouse) ${ }^{v}$} & \\
\hline & $\mathbf{R}$ & MR & MS & $S$ & \\
\hline Diploid & 15 & 24 & 26 & 35 & 46 \\
\hline Tetraploid & 21 & $\begin{array}{c}3 \\
P=0.054\end{array}$ & 26 & 50 & 34 \\
\hline
\end{tabular}

Table 4. Distribution of $\mathbf{5 7 5}$ daylily cultivars by bloom season among ratings for daylily rust severity in a survey in a southern Mississippi landscape in Aug. 2013.

\begin{tabular}{lcccc}
\hline & \multicolumn{3}{c}{ Cultivars (\% by median rating) } & \\
\cline { 2 - 4 } Bloom season $^{\mathbf{z}}$ & $\mathbf{1}$ or $\mathbf{1 . 5}$ & $\mathbf{2}$ & $\mathbf{2 . 5}$ or $\mathbf{3}$ & Cultivars in survey (no.) \\
\hline Extra early & 20 & 45 & 35 & 20 \\
Early & 15 & 42 & 43 & 79 \\
Early midseason & 14 & 40 & 46 & 197 \\
Midseason & 25 & 39 & 36 & 235 \\
Late midseason & 38 & 43 & 19 & 37 \\
Late & 33 & 33 & 33 & 6 \\
Very late & 100 & 0 & 0 & 1 \\
& \multicolumn{2}{c}{ Correlation } & $P=0.001$ & \\
\hline
\end{tabular}

${ }^{2}$ Bloom season according to the American Hemerocallis Society (2015).

$\mathrm{y} 1=$ no or little visual sign of infections; $2=$ moderate infection; $3=$ severe infection.

${ }^{\mathrm{x}}$ Cochran-Mantel-Haenszel test for nonzero correlation (bloom season as an ordinal variable).

wCochran-Mantel-Haenszel test for differing row mean scores (bloom season as a nominal variable). 
Table 5. Distribution of $\mathbf{5 7 5}$ daylily cultivars by winter foliage among ratings for daylily rust severity in a survey in a southern Mississippi landscape in Aug. 2013.

\begin{tabular}{lcccc}
\hline & \multicolumn{2}{c}{ Cultivars (\% by median rating) } & \\
\cline { 2 - 4 } Winter foliage $^{\mathrm{y}}$ & $\mathbf{1}$ or $\mathbf{1 . 5}$ & $\mathbf{2}$ & $\mathbf{2 . 5}$ or $\mathbf{3}$ & Cultivars in survey (no.) \\
\hline Dormant & 31 & 38 & 31 & 75 \\
Semi-evergreen & 17 & 38 & 45 & 248 \\
Evergreen & 21 & 43 & 36 & 252 \\
& \multicolumn{2}{c}{ Correlation $^{\mathrm{x}}: P=0.6836$} & \\
& Mean scores difference & \\
&
\end{tabular}

${ }^{2}$ Winter foliage according to the American Hemerocallis Society (2015).

${ }^{y} 1=$ no or little visual sign of infections; $2=$ moderate infection; $3=$ severe infection

Cochran-Mantel-Haenszel test for nonzero correlation (winter foliage as an ordinal variable).

${ }^{\mathrm{w}}$ Cochran-Mantel-Haenszel test for differing row mean scores (winter foliage as a nominal variable).

inoculated in a greenhouse, $17 \%$ were classified as resistant, $15 \%$ as moderately resistant, $26 \%$ as moderately susceptible, and $42 \%$ as fully susceptible. In a survey of rust severity conducted with nursery field-grown plants in Arkansas in 2001 and 2002 (Robbins, 2003; Robbins and Vann, 2002 ), $75 \%$ and $70 \%$, respectively, of the cultivars examined in each year received ratings of $\leq 2$ (out of 5 ) and were recommended for breeders and consumers. Ratings of $\geq 4$ were received by $4 \%$ and $16 \%$ of the cultivars in 2011 and 2012, respectively; thus, Robbins and Vann (2002) recommended that these cultivars should best be avoided. Intermediate ratings ( $>2$ and $<4$ ) were received by $21 \%$ and $14 \%$ of the cultivars in 2001 and 2002 , respectively. A total of 500 cultivars were examined in each year, and the majority $(>75 \%)$ were rated in both years. None of the cultivars reported in the current survey in Mississippi were included in the aforementioned studies in Georgia and Arkansas. Results of the current survey of daylily cultivars in a Mississippi landscape under conditions favorable to daylily rust are more in line with results reported by Williams-Woodward and Buck (2001) and Mueller et al. (2003) using inoculated plants in a greenhouse in Georgia, whereas percentages of nursery field-grown cultivars in Arkansas reported by Robbins and Vann (2002) and Robbins (2003) as being very resistant to moderately resistant appear to be higher. Environmental conditions in Arkansas may have been less favorable for daylily rust and the authors did not state if fungicides were used in the growing seasons before the two surveys or discuss the likelihood that all plants had been exposed to inoculum.

In exploring relationships between daylily cultivar characteristics and severity of daylily rust in this study, the tendency for tetraploid cultivars to be associated with greater severity of daylily rust than diploid cultivars was of particular interest (Table 3). This association was also noted with cultivars surveyed in 2001 and 2002 in Arkansas (Robbins, 2003; Robbins and Vann, 2002) and cultivars examined in a greenhouse study in Georgia (Mueller et al., 2003) based on our analysis (Table 3). Also, there was a tendency for earlier-blooming cultivars in this survey to have higher ratings for disease severity than later-blooming cultivars (Table 4) and a tendency for a greater percentage of cultivars with dormant winter foliage to have lower disease ratings than cultivars with semievergreen or evergreen foliage (Table 5); however, such associations were not seen with cultivars in the aforementioned surveys in Georgia or Arkansas based on our analyses (data not presented). Such associations may be of interest to other investigators in future studies.

\section{Literature cited}

American Hemerocallis Society. 2015. The American Hemerocallis Society online daylily database. 9 Mar. 2015. <http:// www.daylilies.org/DaylilyDB/>.

Buck, J.W. 2013. Identification of pathotypes in the daylily rust pathogen Puccinia hemerocallidis. J. Phytopathol. 161:784790 .
Buck, J.W. and Y. Ono. 2012. Daylily rust. Plant Health Instructor. doi: 10.1094/ PHI-I-2012-0516-01.

Hernández, J.R., M.E. Palm, and L.A. Castlebury. 2002. Puccinia hemerocallidis, cause of daylily rust, a newly introduced disease in the Americas. Plant Dis. 86: 1194-1198.

Hill, L. and N. Hill. 1991. Daylilies: The perfect perennial. Storey Books, Pownal, VT.

Li, Y.H., M.T. Windham, R.N. Trigiano, D.C. Fare, J.M. Spiers, and W.E. Copes. 2007. Microscopic and macroscopic studies of the development of Puccinia hemerocallidis in resistant and susceptible daylily cultivars. Plant Dis. 91:664-668.

Mueller, D.S. and J.W. Buck. 2003. Effects of light, temperature, and leaf wetness duration on daylily rust. Plant Dis. $87: 442-445$.

Mueller, D.S., J.L. Williams-Woodward, and J.W. Buck. 2003. Resistance of daylily cultivars to the daylily rust pathogen, Puccinia hemerocallidis. HortScience 38:11371140.

Pollard, A.N., P.C. Coggins, and P.R. Knight. 2004. Sensory evaluation of edible daylilies (Hemerocallis sp.). HortScience 39:783 (abstr.).

Robbins, J. 2003. Daylilies database. 15 Mar. 2015. <http://daylilies.uaex.edu/>.

Robbins, J.A. and S. Vann. 2002. Susceptibility of daylily to daylily rust in Arkansas, p. 86-91. In: J.R. Clark and M.R. Evans (eds.). Horticultural studies 2001. Arkansas Agr. Expt. Sta., Res. Ser. 494.

Stout, A.B. 1986. Daylilies: The wild species and garden clones, both old and new, of the genus Hemerocallis. Sagapress, Millwood, NY.

Weather Underground. 2013. Weather history for KHBG. 15 Mar. 2015. <http:// www.wunderground.com/history/ airport/KHBG/2013/8/31/ MonthlyHistory.html .

Williams-Woodward, J.L. and J.W. Buck. 2001. Daylily rust caused by Puccinia hemerocallidis: A new disease on daylily in the United States. Proc. Southern Nursery Assn. Res. Conf. 46:234-236.

Williams-Woodward, J.L., J.F. Hennen, K.W. Parda, and J.M. Fowler. 2001. First report of daylily rust in the United States. Plant Dis. 85:1121. 